\title{
Enquête sur les photomontages politiques de Marinus Jacob Kjeldgaard (1932-1940)
}

\section{Gunner Byskov}

Traducteur : Marc Auchet

\section{OpenEdition}

\section{Journals}

Édition électronique

URL : http://journals.openedition.org/genesis/1454

DOI : 10.4000/genesis. 1454

ISSN : 2268-1590

\section{Éditeur :}

Presses universitaires de Paris Sorbonne (PUPS), Société internationale de génétique artistique littéraire et scientifique (SIGALES)

\section{Édition imprimée}

Date de publication : 15 avril 2015

Pagination : 51-66

ISBN : 978-2-84050-992-9

ISSN : 1167-5101

Référence électronique

Gunner Byskov, «Enquête sur les photomontages politiques de Marinus Jacob Kjeldgaard

(1932-1940) », Genesis [En ligne], 40 | 2015, mis en ligne le 28 mars 2017, consulté le 17 mai 2019.

URL : http://journals.openedition.org/genesis/1454; DOI : 10.4000/genesis.1454 


\section{Enquête sur les photomontages politiques de Marinus Jacob Kjeldgaard (1932-1940)}

Gunner Byskov

\section{Prologue}

L

e vendredi 14 novembre 2003, j'étais au Carrousel du Louvre, à l'occasion de Paris Photo, cette grande exposition à laquelle participent des galeries de photos du monde entier. Sur le stand numéro D12, le galeriste parisien Bernard Dudoignon exposait dix montages photographiques dont je n'avais jamais entendu parler. Ils dataient des années trente et leur qualité était telle qu'ils me coupèrent le souffle et piquèrent ma curiosité.

Il était évident que ces photomontages avaient été conçus comme des commentaires à chaud des événements politiques de la fin des années trente. Hitler figurait sur la plupart d'entre eux, de même que Mussolini, Franco, Daladier, Roosevelt et d'autres. Ils étaient très expressifs, et lorsqu'on se rapprochait, comme on le fait d'habitude, pour voir qui avait bien pu être leur auteur, on découvrait une petite pancarte avec le nom « Marinus Jacob Kjeldgaard ».

Marinus Jacob Kjeldgaard? De qui pouvait-il s'agir? Bernard Dudoignon ne savait rien de lui hormis qu'il était Danois. Dudoignon avait fait réaliser une petite brochure intitulée Rien que la terre, où on pouvait lire ce qu'on savait de Marinus : né le 4 septembre 1884, il a habité Paris, au 14 de la rue Saint-Louis-en-l'Île, et est mort le 6 février 1964. Le reste ne nous est pas connu.

Et les montages ? Nous connaissons les pathétiques combinaisons de négatifs de Rejlander et Robinson, les collages de dentelle dadaïstes de Hannah Höch, et surtout les premières pages du journal ouvrier Arbeiter Illustrierte Zeitung (AIZ) de John Heartfield, engagé dans la lutte contre le nazisme. Mais ces photomontages étaient différents.

Ils étaient élégants, subtils, techniquement parfaits et n'avaient rien de la rhétorique quelque peu tapageuse de John Heartfield. Leur niveau d'analyse était élevé et ils

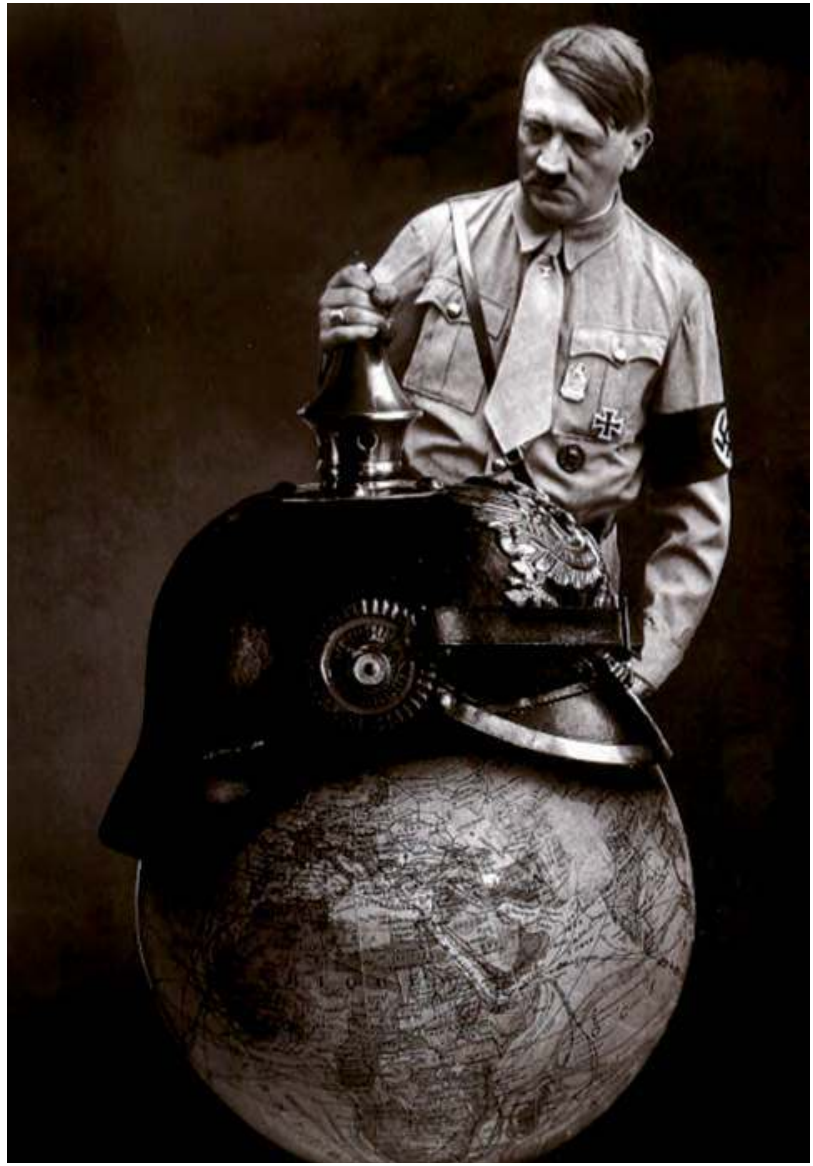

Fig. 1 : Marinus Jacob Kjeldgaard, « Rien que la terre » Photomontage original $(35,5 \times 25,5 \mathrm{~cm})$, imprimé dans Marianne, $\mathrm{n}^{\circ} 360$ le 13 septembre $1939(28,4 \times 21 \mathrm{~cm})$

témoignaient d'une compréhension claire de la personne d'Hitler, de son agression, sa stratégie et son objectif final : la guerre et la domination du monde. 
On se rendait facilement compte qu'ils avaient été utilisés dans la presse quotidienne, comme on le fait souvent avec les dessins, mais la photographie leur donnait un caractère d'authenticité très particulier, que le dessin est incapable de communiquer.

En plus de cela, il était difficile de voir qu'il s'agissait de montages. Leur qualité était irréprochable ; il était évident qu'il s'agissait d'un homme qui, non seulement s'y connaissait en photographie, mais comprenait de quoi il retournait. La question se posait, lancinante : qui était cet homme et d'où venaient ces images ?

C'est ainsi que commença la chasse à Marinus, cet inconnu ayant vécu à Paris, entouré de mystère, et apparemment l'auteur de ces dix images extraordinaires.

\section{Qui est Marinus ?}

\section{L'identification}

La brochure Rien que la terre attribue à Marinus Jacob Kjeldgaard l'honneur d'avoir réalisé les montages de la revue littéraire et politique Marianne - mais comment en être sûr ? Comme il collaborait régulièrement au journal, ses contributions n'étaient pas identifiées et son nom n'est cité qu'une seule fois, dans le numéro 33 publié le 7 juin 1933, en relation avec un montage. Cela s'explique par le fait qu'il avait fourni l'une des photographies qui avaient servi à sa réalisation.

Au dos d'un grand nombre des tirages existants, on trouve toutefois un tampon avec le nom de Marinus, son adresse (fig. 2), mais aussi à plusieurs occasions les œuvres qui ont été reproduites dans d'autres publications, par exemple Photography Year Book 1935 et Life Magazine du 19 décembre 1938, avec la mention désignant explicitement Marinus comme leur auteur.

Dans une lettre privée, Marinus indique d'ailleurs luimême qu'il était un collaborateur régulier de l'hebdomadaire Marianne, et, dans la livraison du mois de mars 1940 de The Strand Magazine, figure un article signé H. J. Greenwall de plus de onze pages consacré exclusivement à Marinus et illustré de onze de ses montages. En plus de tout cela, après avoir comparé une grande quantité de notes écrites à la main au dos des images avec des lettres privées adressées à sa famille au Danemark, un graphologue conclut avec une probabilité proche de la certitude, que toutes les pièces mentionnées ont été écrites de la même main.
Tous ces éléments permettent d'affirmer que Marinus Jacob Kjeldgaard est l'auteur des montages publiés par Marianne de 1932 à 1940.

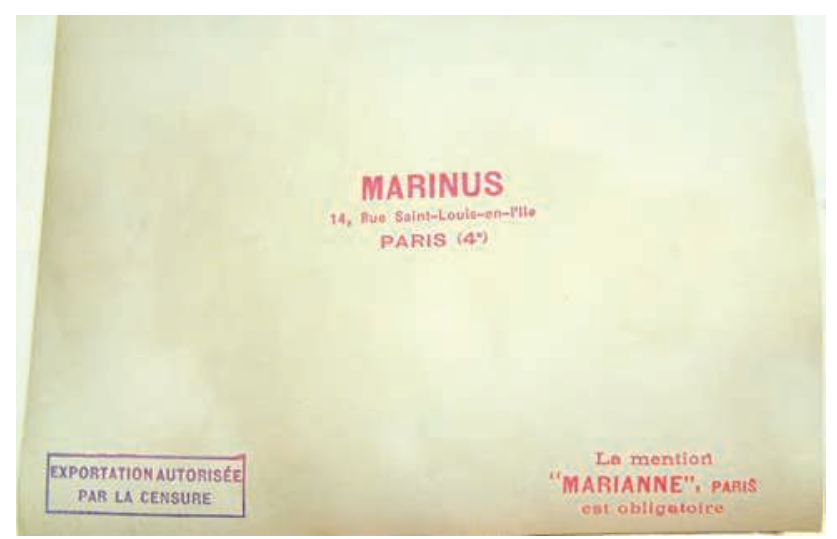

Fig. 2 : Tampons figurant au dos du montage publié le 6 décembre 1939 dans Marianne $\mathrm{n}^{\circ} 372$, représentant Hermann Göring et Joachim von Ribbentrop, tous deux naufragés mais tentant chacun de frapper l'autre avec une bouteille de Champagne marquées, l'une d'une croix gammée, la seconde d'une faucille

\section{L'histoire de Marinus en bref}

Après avoir terminé un apprentissage de peintre en bâtiment à Copenhague et suivi les cours de l'Académie des beaux-arts (Kunstakademiet) pendant cinq ans, il s'installe à Paris en 1909. Il ne retournera jamais au Danemark.

À Paris, il se débrouille d'abord du mieux qu'il peut avant d'être engagé par l'hebdomadaire $J$ 'ai $v u$, vraisemblablement en 1916. Au cours des années vingt, il se présente comme correspondant et journaliste. Il travaille sans doute pour les éditions Gallimard à partir de 1931, pour l'hebdomadaire Voilà et, mis à part quelques interruptions, est rattaché à Marianne de 1932 à 1940.

Après la guerre, il ouvre un atelier de photographie rue Froideveaux, puis une petite agence de photographie. Il meurt en 1964.

On a de multiples raisons de penser que ses montages étaient assez connus en France, en particulier ceux de la période allant de 1938 à 1940. Alexander Liberman mentionne son nom et s'est inspiré de lui, et le journaliste britannique H. J. Greenwall le cite dans ses mémoires (When France Fell, 1958). Mais, en tant que personne, il semble avoir eu une vie tranquille et extrêmement discrète. Il n'avait pas de famille et l'on a le sentiment qu'il avait très peu de contacts à la fin de sa vie. 
Comme le tirage moyen de Marianne était de soixante mille exemplaires, ses « unes » étaient très connues, elles ne semblent pas néanmoins avoir exercé une influence notable sur la vie politique. Marinus et Marianne se situaient d'ailleurs en dehors de l'antagonisme de plus en plus marqué opposant les formations politiques les plus représentatives. Ils ne soutenaient ni le Front populaire, ni les partis conservateurs traditionnels. Ils critiquaient Staline aussi bien qu'Hitler, informèrent les lecteurs au cours des années trente du danger que représentaient Hitler et, à partir du mois d'août 1939, Staline - quand une majorité de Français auraient préféré l'ignorer.

\section{Marinus et le Danemark}

Marinus n'avait pratiquement aucun lien avec le Danemark et l'on ne trouve que des traces très indirectes de son pays d'origine dans ses travaux. Le montage qui figure en première page de la livraison du 6 mars 1940 de Marianne, en légende «L'épouvantail des neutres », peut être considéré comme une salutation adressée au Danemark, qui était neutre à l'époque et avait si peur d'Hitler qu'il souhaitait bien le rester.

Un mois plus tard, le 9 avril 1940, l'Allemagne envahit pourtant le Danemark, qui choisit la voie d'une collaboration étroite avec l'occupant. Ces deux pays ne furent par conséquent jamais en guerre l'un avec l'autre à cette période et le Danemark est le pays d'Europe ayant subi le moins de dommages pendant la Seconde Guerre mondiale.

Pendant toute une période, le reste de l'Europe a pu considérer que le Danemark était du côté de l'Allemagne, mais, au cours des dernières années de guerre - de mauvaises langues prétendent que ce fut à partir du moment où la défaite de l'Allemagne sembla inéluctable - la résistance s'organisa petit à petit. Les mêmes mauvaises langues parlent d' « entrée en guerre rétroactive », qui permit au Danemark de se retrouver du côté des vainqueurs lors du traité de paix de 1945 .

\section{Réflexions méthodologiques}

\section{Le détective-exemple 1}

En novembre 2003, date de ma « découverte », une réflexion méthodologique ne s'était pas encore imposée : on se trouvait simplement face à un grand point d'interrogation.
Rapidement, on en arriva à la conclusion que l'approche méthodologique devait s'inspirer des méthodes simples utilisées par les détectives. Il y avait un homme né au Danemark, il avait un nom et une date de naissance : où pouvait-on retrouver ses traces?

Il fallait suivre toutes les pistes susceptibles de fournir des renseignements et, en 2003, il était évident que la recherche concernant Marinus devait commencer sur Internet. Les premières investigations ne donnèrent aucun résultat, mis à part - comme on pouvait s'y attendre des indications concernant la galerie de l'exposition Paris Photo.

Il s'avéra qu'il était plus judicieux de diriger la recherche vers Marianne. Les sites Internet ne fournissent pas beaucoup d'informations et le journal avait apparemment fait l'objet d'une seule publication, dans le livre La Gauche hebdomadaire de Claude Estier en 1962. Mais je découvris que, pour des raisons inconnues, la Statsbibliotek (Bibliothèque nationale) d'Aarhus (Danemark), où j'habitais, possédait tous les numéros de Marianne, du numéro 1 au numéro 327 de janvier 1939. L'abonnement avait été pris dès le premier jour, si bien que la Statsbibliotek disposait d'une collection unique, qui, à ma connaissance, n'a d'équivalent qu'à la Bibliothèque nationale de France, la Royal Library de Londres et la Bibliothèque universitaire de Genève.

J'avais ainsi accès aux trois cent vingt-sept numéros du journal et je pus très rapidement me faire une idée d'ensemble des montages de Marianne - mais la personne de Marinus restait entourée de mystère.

Le nom Kjeldgaard est très connu au Danemark, bien qu'il ne soit pas particulièrement répandu. Utilisant les cabinets de recherche généalogique danois, puis les sites Internet, j'envoyai des courriers à tous les Kjeldgaard ayant une adresse électronique. Deux semaines plus tard, je reçus une réponse positive. Un certain Jack Kjeldgaard aux États-Unis me disait que Marinus Jacob Kjeldgaard était le cousin de son grand-père. Il était connu comme artiste peintre. L'arbre généalogique de Jack Kjeldgaard révélait que Marinus était le cinquième d'une fratrie de sept frères et qu'il avait deux neveux. Je contactai l'un d'entre eux. J'appris qu'il était professeur émérite en biologie moléculaire et - quelle surprise ! - qu'il n'habitait pas à plus de trois kilomètres de chez moi ! Il était disposé à 
me raconter ce qu'il savait de son oncle Marinus, à qui il avait rendu visite à Paris et qu'il avait même pris en photo lorsqu'il faisait des recherches à l'Institut Pasteur en 1957.

Et les choses continuèrent ainsi. Dans les archives locales et nationales, les bibliothèques de Paris et de Copenhague, je découvris, un par un, des indices. Lentement une image de Marinus Jacob Kjeldgaard commença à se former. Dans son principe, les méthodes que j'employai rappelait celles de Sherlock Holmes, d'Hercule Poirot, de Maigret. Il fallait reconstituer un puzzle. Le raisonnement et l'intuition étaient tous deux nécessaires. Une pièce pouvait mener à la suivante, mais beaucoup de pièces restèrent longtemps isolées, sans voisines. Et pourtant, d'autres pièces vinrent tranquillement s'ajouter aux précédentes. Peu à peu, elles offrirent l'image d'un homme né à Copenhague, jusqu'à sa mort solitaire à Créteil, soixante-dix-neuf années plus tard.

\section{Le détective - exemple 2}

Aux Archives nationales de Copenhague, je consultai le recensement de 1885 (Marinus Jacob Kjeldgaard avait moins d'un an), le registre paroissial, ses formulaires d'inscription à l'école.

Mais pour moi, le plus important fut sa fiche d'enregistrement qui, selon les règles en vigueur à l'époque, était tenue à jour par la police. Elle commençait à la date du 1er mai 1898, c'est-à-dire juste avant le quatorzième anniversaire de Marinus. Ses changements d'adresses y étaient mentionnés jusqu'au 1er novembre 1909, où figure la mention « radié ».

La première preuve officielle de sa présence en France date du recensement de 1926, où il figure en tant que célibataire et journaliste à l'adresse 20, rue Ernest-Cresson, à Paris.

\section{Le détective - exemple 3}

Lorsque Marinus est mort, il était encore citoyen danois, et il se peut que le consulat général de Paris ait été contacté lors de son décès. Aux Archives nationales a été consignée une affaire datant de 1964-1965 enregistrée sous le numéro 35.M.4673. Avec Niels Ole Kjeldgaard, le neveu, nous ouvrîmes en février 2004 la caisse qui renfermait les papiers officiels.
Il y avait des télégrammes échangés à l'occasion du décès, des lettres destinées au règlement notarial de la succession. Mais il y avait aussi un rapport du consul danois, Aage Ankersen, qui, d'après l'ami de Marinus, l'avocat Hamet, l'avait informé du décès, s'était rendu dans l'arrièrecour du 14, rue Saint-Louis-en-l'Île et avait apposé des scellés. Dans son rapport, le consul décrit l'appartement, sale, en désordre, plein de papiers et d'objets divers, et le concierge raconte que Marinus « était en contact avec plusieurs maisons d'édition au sujet de la livraison de couvertures de livres en couleurs ».

Le règlement de la succession montre que tout l'inventaire a été vendu par le commisaire-priseur, M. Thuillier, pour la somme de 474,05 francs - mais l'acheteur n'est pas mentionné. Ainsi se perd la trace des biens ayant appartenu à Marinus.

\section{Le détective-exemple 4}

Après avoir mené cette enquête à Paris, j'ai eu la chance inouïe d'être aidé par l'historienne d'art Marie-Hélène Joyeux, et comme je souhaitais lui montrer un montage original de Marinus, nous rendîmes visite à un galeriste qui en possédait deux. Il nous expliqua comment il les avait dénichés et lorsque nous insistâmes pour qu'il nous oriente vers d'autres pistes possibles, il se souvint qu'il avait entendu parler d'un collectionneur américain qui avait quelques montages qui « faisaient penser à ceux de Heartfield ». Le collectionneur s'était installé en Angleterre en 1981 ; il était mort entre-temps, mais sa veuve habitait sans doute encore quelque part là-bas. La piste était ténue, mais le galeriste pensait que Philip Garner de chez Christie's, à Londres, connaissait peut-être cette dame.

J'eus la chance de pouvoir le joindre par téléphone et il me confirma qu'il la connaissait. Il me promit qu'il prendrait contact avec elle pour lui demander si elle était toujours en possession de ces montages. Il me donna ensuite le numéro de téléphone de la veuve ainsi que la permission de l'appeler. J'avoue que l'instant où j'entrai en contact avec elle fut un grand moment. Elle m'apprit qu'elle possédait six montages originaux de Marinus, dont l'un n'avait jamais été imprimé et était inconnu. Sherlock Holmes ressentait certainement la même chose que moi quand la solution de l'énigme se révélait brusquement à lui. 


\section{Le lecteur contextuel}

\section{Exemple 1 : le 11 octobre 1933 (fig. 3)}

Pour un Danois qui n'est pas spécialiste de la politique française des années trente, il est nécessaire de beaucoup travailler pour comprendre les situations que les montages commentaient, ainsi que leur sens profond, souvent exprimé avec élégance. Je dus lire les principaux articles politiques de Marianne pour savoir quels événements avaient marqué la politique (internationale) à cette époque et surtout quelle position le journal avait prise à leur égard.

C'était d'autant plus difficile que Marianne n'avait pas d'éditoriaux. En réalité, on peut dire que les grands montages photographiques de Marinus, qui figuraient en première page, faisaient office d'éditoriaux, mais ils n'étaient pas d'une grande aide pour comprendre de quoi il retournait. Comme je n'avais pas la possibilité, pour toutes sortes de raisons, de consulter des sources de première main, je puisai les informations les plus importantes dans des ouvrages qui traitaient de cette période, et, bien sûr, en ayant recours à Internet.

Les résultats que l'on obtient par ce genre de recherche - y compris dans les livres, d'ailleurs - ne sont pas toujours fiables, si bien que je décidai de ne tenir compte que des récits des divers événements ayant été rapportés au moins

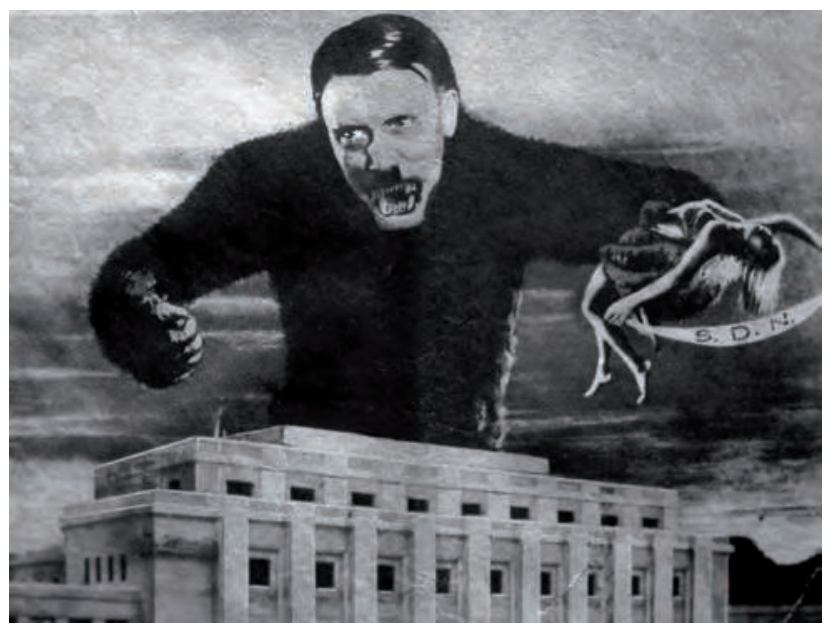

Fig. 3 : Marinus Jacob Kjeldgaard, « Le King-Kong européen n’enlèvera-t-il pas la pauvre S.D.N. avant qu'elle ait pu entrer dans son nouveau palais de Genève ? » (Marianne, 11 octobre 1933) à deux endroits différents et dans les mêmes termes. En cas de doutes, je consultai des historiens et des spécialistes de la langue française.

Une fois que je m'étais fait une idée de ce qui avait pu se passer au moment de la publication du journal, il n'y avait plus qu'à se lancer dans l'analyse contextuelle. Prenons un exemple.

Le montage de la une de Marianne du 11 octobre 1933 présente Hitler sous les traits d'un gigantesque singe qui se tient derrière un grand palais (le bâtiment de la Société des Nations), serrant une jeune fille dans sa main. La fille tient un torchon sur lequel sont écrites les initiales «S.D.N. », et sous le montage on peut lire la légende suivante : «Le King-Kong européen n'enlèvera-t-il pas la pauvre S.D.N. avant qu'elle ait pu entrer dans son nouveau palais de Genève ? »

Cette fois, Marinus a pris comme point de départ une image tirée de l'actualité. La première du film King-Kong avait eu lieu à Paris le 29 septembre 1933, si bien que tout le monde connaissait l'image du singe gigantesque. Sur l'affiche du film, la fille désespérée retenue prisonnière dans la main du singe n'a pas de torchon (fig. 4), c'est donc quelque chose que Marinus a ajouté, et le sigle S.D.N. se rapporte à la Société des Nations. Tout ceci permet de comprendre et de décrire le sens de ce montage.

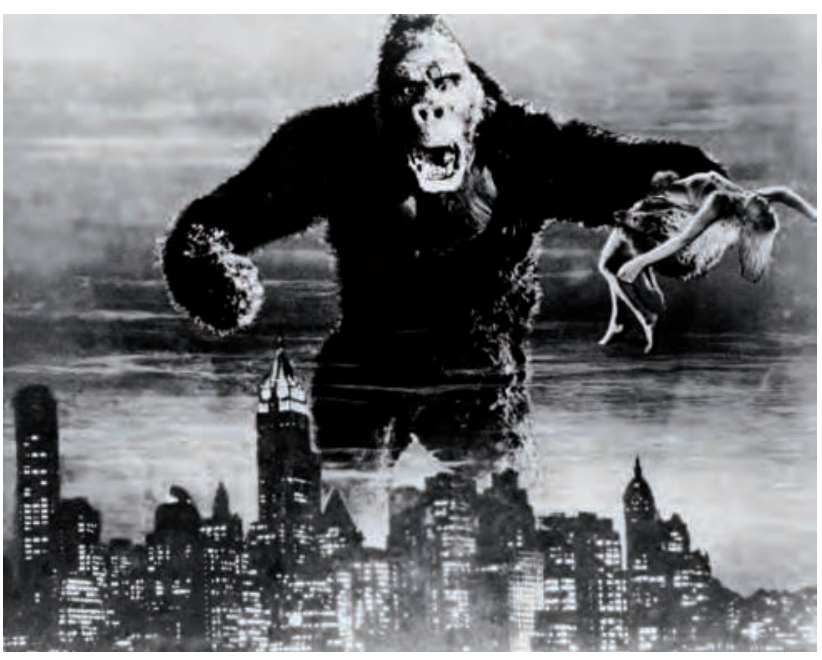

Fig. 4 


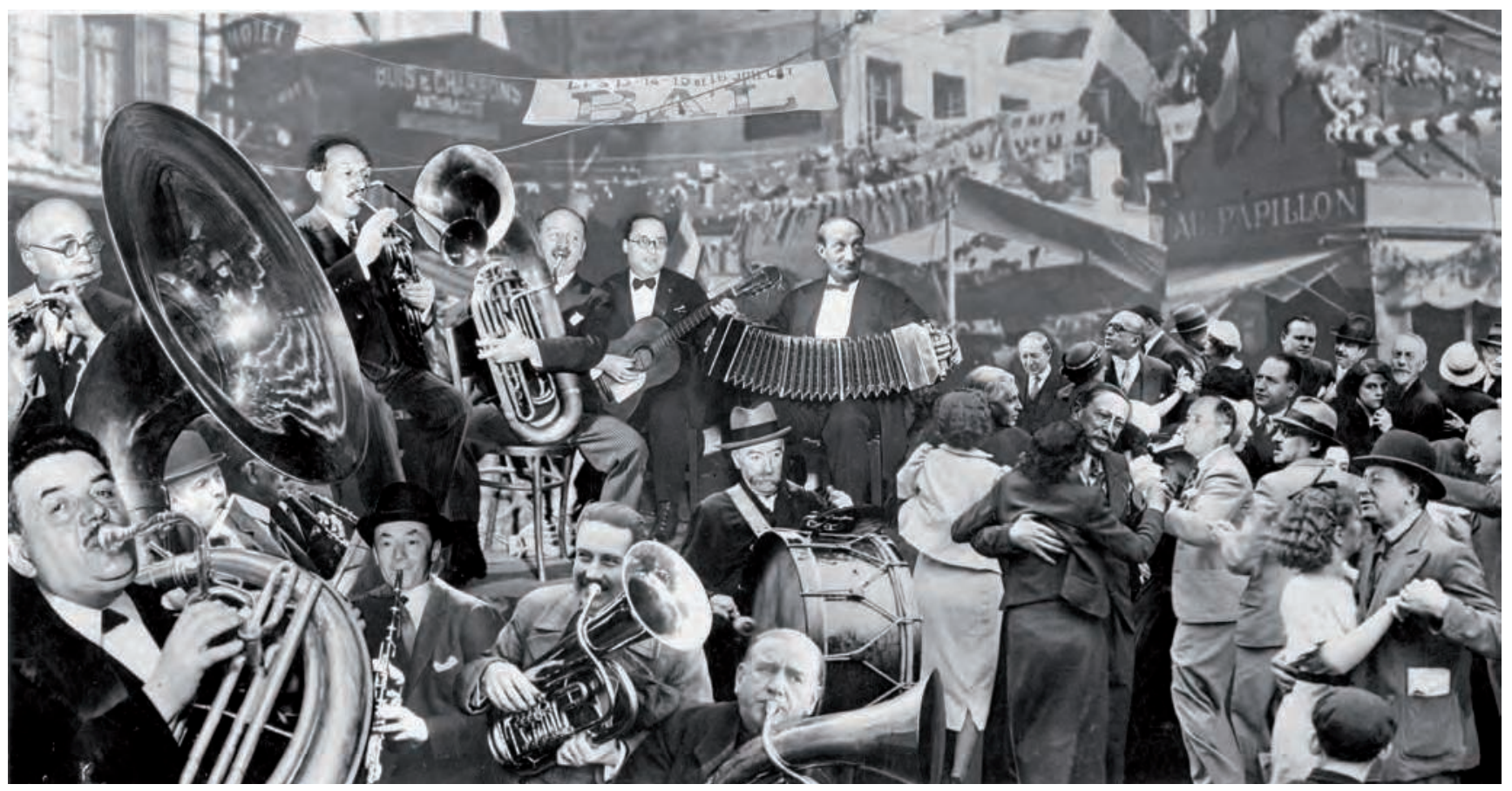

Depuis février 1933, la SDN réunie à Genève mène de difficiles négociations sur le désarmement, dans le but de créer la détente en Europe, et est par ailleurs sur le point d'emménager dans le nouveau quartier général de l'organisation, le Palais des Nations, en construction depuis 1929. Mais il est évident pour beaucoup qu'Hitler joue un double jeu, ce qui inspire à Marinus et à Marianne l'idée de ce montage qui est plus qu'une simple allusion au fait qu'Hitler avait l'intention de bloquer le travail de la SDN - avant même que l'organisation ait pris possession du nouveau palais. En représentant celleci sous les traits d'une femme - jolie au demeurant entièrement soumise aux caprices du monstre Hitler, cette « une » dénonce la faiblesse de la SDN.

C'est un montage prophétique, car trois jours après sa publication en première page de Marianne, Hitler fait savoir que l'Allemagne se retire de la SDN, ce qui permet aux Allemands de se concentrer sur leur effort d'armement en toute tranquillité. On put ainsi constater que la SDN était précisément aussi faible que le montage l'avait prédit.

Nous avons des raisons de penser que cette livraison du journal a été lue en Allemagne, car trois semaines plus tard, le 3 novembre 1933, le chef des SS et de la police allemande (Reichsführer-SS und Chef der Deutschen Polizei) décréta l'interdiction de Marianne en Allemagne, interdiction qui, par la suite, fut étendue à l'Italie, l'Autriche, la Tchécoslovaquie et au Japon.
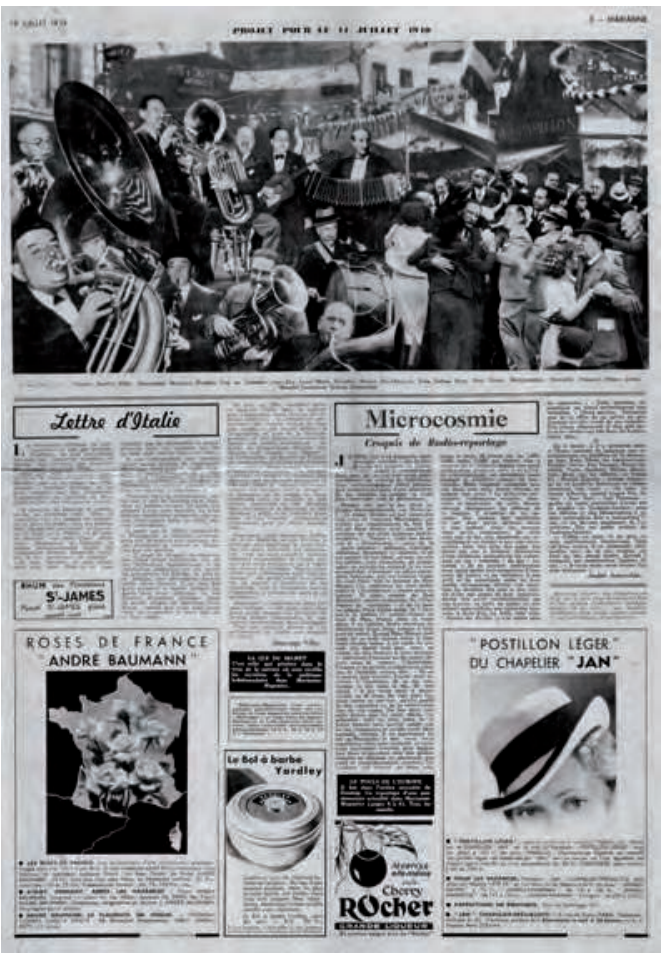

Fig. 5 et 6 : Marinus Jacob Kjeldgaard, « Projet pour le 14 juillet 1940 ». Dans ce photomontage $(15 \times 29,4 \mathrm{~cm})$ publié dans Marianne $\mathrm{n}^{\circ} 352 \mathrm{du}$ 19 juillet $1939(18 \times 34,9 \mathrm{~cm})$, on reconnaît notamment Édouard Herriot, Paul Reynaud, Jean Zay, Louis Marin, Édouard Daladier, Léon Blum, Maurice Thorez, Pierre Laval, Georges Mandel, Jules Jeanneney, Albert Lebrun (copie de photo d'après l'original) 


\section{Exemple 2 : le 19 juillet 1939 ((fig. 5 et 6$)$}

Ici, le peuple est en liesse ! On fête le 14 juillet, tout le monde est dehors, les hommes politiques de tous bords jouent et dansent tous ensemble, sans tenir compte des divergences et des désaccords qui les opposent en temps normal. Mais cette image a quelque chose de particulier : elle est accompagnée de la légende : «Projet pour le 14 juillet 1940 ». Elle ne décrit pas la fête de l'année 1939, mais le rêve de la population pour l'avenir.

L'été 1939, l'atmosphère est lourde en France. On a pris conscience du fait que la guerre est plus menaçante que jamais. Après s'être emparé sans combat de l'Autriche et de la Tchécoslovaquie, Hitler s'en prend à la Pologne ; la France et l'Angleterre sont fermement déterminées à l'arrêter.

Quand la plupart des esprits sont en proie à la peur, Marinus exprime l'espoir que la grande catastrophe ne frappera pas le pays et que l'on pourra une nouvelle fois fêter le 14 juillet en 1940. Il y a de quoi être ému, surtout si l'on sait qu'un an plus tard il n'y eut aucune raison de faire la fête, car à ce moment-là l'armée française, que les Français considéraient comme la plus forte au monde, avait été balayée en l'espace d'un mois et les Allemands étaient à Paris. La fête du 14 juillet fut supprimée.

\section{Exemple 3 : le 23 août 1939 (fig. 7)}

L'alliance entre la Russie communiste et l'Allemagne nazie a été pendant longtemps le pire cauchemar des diplomates occidentaux et, le 23 août 1939, ce cauchemar devient réalité quand Molotov et von Ribbentrop signent le pacte de non-agression entre les deux pays. Même si on ne connait pas toutes les clauses de ce pacte, chacun sait que la guerre est désormais inévitable.

Pour une fois, ce ne sont pas Hitler, Goebbels ou Göring qui poussent à la roue. Ce sont Roosevelt et Staline, tandis que Chamberlain et Daladier montent la garde en arborant un grand sourire, sans intervenir. Les deux chefs d'État laissent les choses se faire, naïvement, sans s'inquiéter, et ce sont les États-Unis, avec leur parti pris non interventionniste, et Staline, avec sa nouvelle alliance, qui mènent inéluctablement le monde vers la guerre.
Ce montage décrit de façon très subtile les causes de la guerre. Les dirigeants européens avaient depuis longtemps si peur qu'une nouvelle guerre éclate qu'ils avaient naïvement laissé passer beaucoup trop d'occasions, et le président des États-Unis, avec le soutien du peuple américain, avait évité de se mêler de ce qui lui semblait être des affaires internes à l'Europe. Lorsque Staline décide de jouer son propre jeu, la guerre peut commencer.

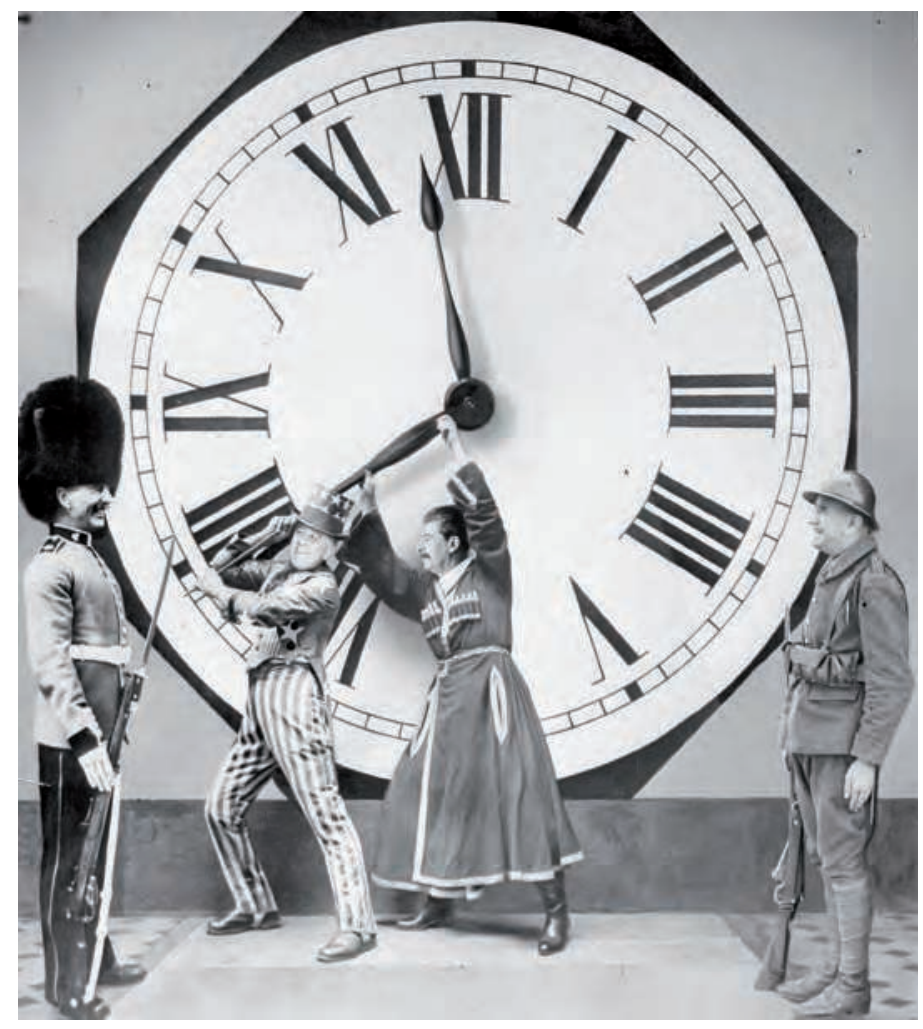

Fig. 7 : Marinus Jacob Kjeldgaard, «L'horloge de la paix. Bientôt moins cinq... » Copie de photo d'après l'original $(25,7 \times 22,7 \mathrm{~cm})$ publié dans Marianne $\mathrm{n}^{\circ} 357,23$ août $1939(22,3 \times 20,6 \mathrm{~cm})$.

De gauche à droite : Neville Chamberlain, Franklin D. Roosevelt, Joseph Staline, Édouard Daladier 


\section{Exemple 4 : le 13 mars 1940 (fig. 8 et 9)}

Exactement comme lorsque le président des États-Unis envoie de nos jours son secrétaire d'État au Moyen-Orient pour promouvoir une nouvelle fois la paix, Roosevelt dépêcha au cours de l'hiver 1940 son représentant pour visiter une série d'États européens, et, bien entendu, l'Allemagne. Cet homme est le sous-secrétaire d'État Sumner Welles. Au début du mois de mars 1940, il rencontre Hitler ; on trouve la phrase suivante dans le rapport qu'il rédige et envoie à cette occasion : "Ses paroles et son comportement donnaient une impression de dignité qui ne correspondaient pas le moins du monde à l'effet comique que créent la moustache et la chevelure dont l'affublent les caricatures » (He was dignified both in speech and movement, and there was not the slightest impression of the comic effect from moustache and hair which one sees in his caricatures). On a des raisons de penser qu'Hitler a charmé l'envoyé de Roosevelt, si bien que rien n'est ressorti de cette entrevue, aucune initiative de paix, strictement rien. Les journaux firent écho à cela, évidemment, et Marinus et Marianne ne purent s'empêcher de dénoncer la naïveté de Sumner Welles, cet Américain qui ne comprenait apparemment rien à rien. Marinus le représente en train d'admirer un Hitler habillé de façon extrêmement étrange, sur le point d'exécuter une cabriole impressionnante, portant une culotte à volants qui lui va jusqu'aux genoux, des chaussures de clown aux pieds (à moins qu'il ne s'agisse de jambes de cheval), et une croix gammée sur la poitrine. Le commentaire de Marianne montre donc Sumner Welles en train de regarder Hitler qui se paie sa tête alors qu'il se prépare à la guerre, et Welles ne se rend compte de rien.

\section{Les processus de création}

\section{La technique de montage}

Life Magazine, dans sa livraison du 19 décembre 1938, a décrit les montages de la façon suivante :

Ce qui rend ces amusantes falsifications tellement exceptionnelles, c'est l'habileté avec laquelle les montages ont été faits. La plupart des photomontages échouent lorsqu'il faut placer correctement sur le cou la tête qu'on a découpée, et faire en
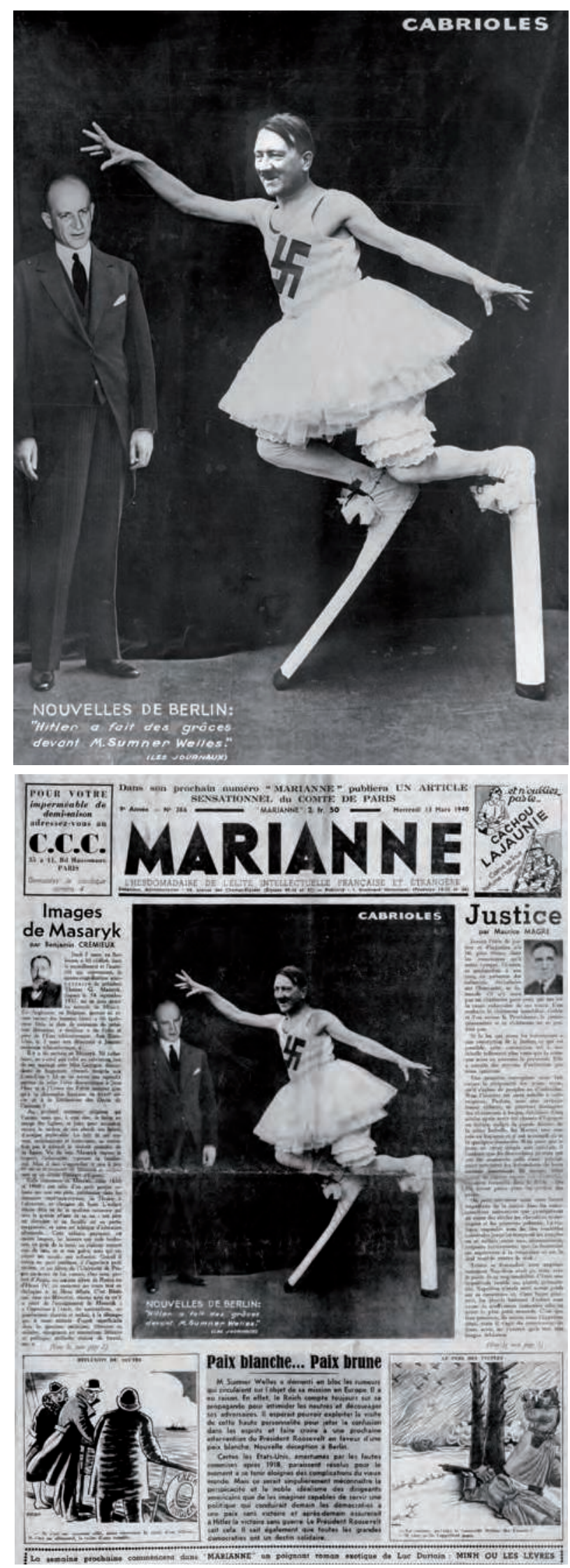

Fig. 8 et 9 : Marinus Jacob Kjeldgaard, « Hitler a fait des grâces devant M. Sumner Welles », copie de photo d'après l'original $(22,8 \times 17 \mathrm{~cm})$, publié dans Marianne $\mathrm{n}^{\circ}$ 386, 13 mars 1940.

Hitler, chaussé comme un clown, exécute une cabriole devant Sumner Welles,vice-ministre des Affaires étrangères des États-Unis 
sorte que les proportions de la tête correspondent à celles du corps. La plupart des personnages qui figurent dans ces pages ont l'air assez naturel. Le technicien de Marianne, Marinus, a passé les archives au peigne fin pour trouver des photos adaptées des célébrités souhaitées, il a copié ces photos en 5 ou 6 formats différents, les a ajustées, travaillées et ajustées encore à l'infini. Et pour finir, il a photographié la composition toute entière, après avoir rassemblé toutes les pièces et l'avoir retouchée avec soin.

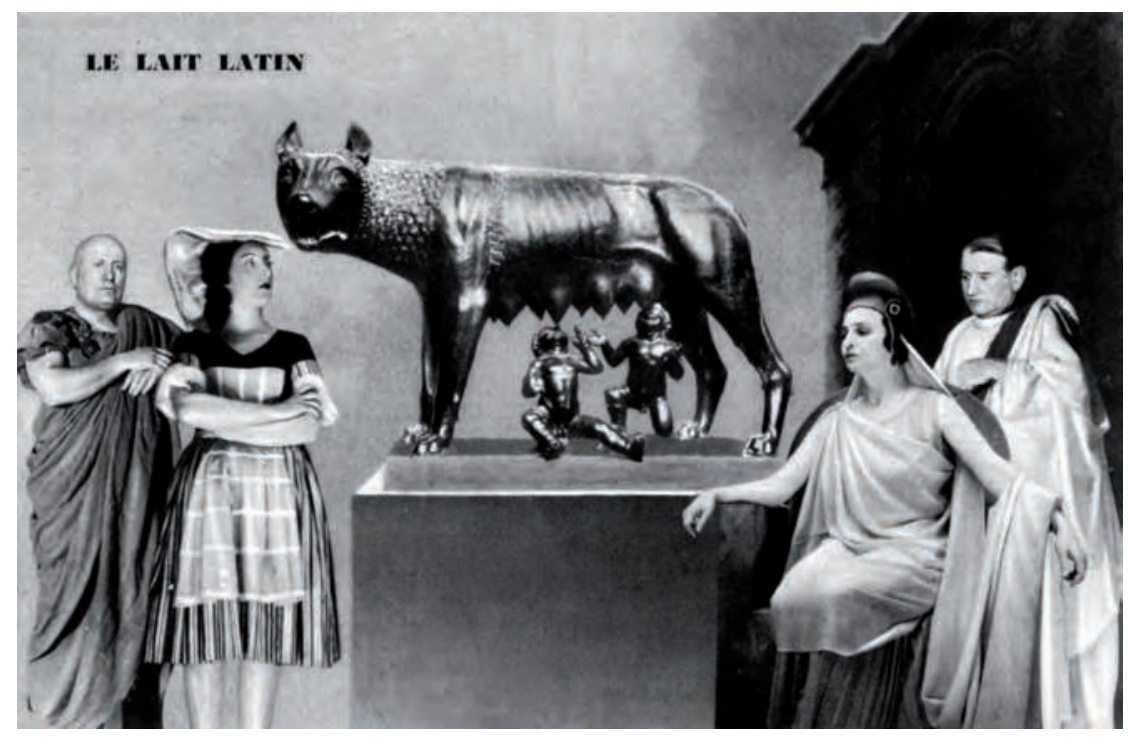

Fig. 10 : Marinus Jacob Kjeldgaard, « Le lait latin »

Copie de la photo originale publiée dans Marianne, 27 juillet 1938 À gauche : Mussolini / À droite : Daladier

Dès le début de sa carrière, avec J'ai vu en 1916-1917, Marinus a voulu réaliser des photomontages ressemblant le plus possible à des photographies authentiques. Cela voulait surtout dire que la perspective devait être correcte et que la lumière devait éclairer de façon similaire tous les personnages de l'image.

Pour cela, il avait constitué de volumineuses archives de photographies d'agence représentant les hommes politiques les plus importants pris sous de nombreux angles, et lorsque le comité de rédaction et lui étaient tombés d'accord sur une idée utilisable, il se mettait à chercher l'arrière-plan et les portraits appropriés. Il explique lui-même dans The Strand Magazine (du mois de mars 1940, p. 457) qu'il était parfois obligé de passer en revue plus de deux mille portraits d'Hitler avant de trouver celui qu'il fallait.

Après avoir sélectionné les portraits qui convenaient quant à l'expression des visages et la perspective, les avoir photographiés et copiés dans différents formats, et corrigé les effets de lumière, le travail d'assemblage du montage pouvait commencer.

Mais il n'avait pas toujours les photos nécessaires dans ses archives, et dans ces cas-là il les réalisait lui-même. Il fallait bien sûr que les visages soient authentiques, mais le reste des personnages pouvait fort bien provenir de modèles - c'était d'ailleurs souvent le cas. Life Magazine cite un exemple tiré du numéro 301 de Marianne, daté du 27 juillet 1938, où figure le montage «Le lait latin » (fig. 10), avec Mussolini et Daladier placés de part et d'autre de la sculpture classique représentant Romulus et Rémus en train de téter une louve. Ici, le corps de Mussolini est celui du factotum de Marianne et le corps de Daladier celui du coursier du journal. C'était assez simple : lorsque Marinus avait trouvé la bonne expression parmi les portraits qu'il avait en archive, il pouvait déguiser les modèles et leur faire prendre les attitudes qui correspondaient au visage, en prenant garde que l'éclairage soit le même que celui des portraits. Il n'y avait alors plus qu'à faire le montage.

Les images retenues étaient ensuite photographiées, les négatifs développés puis tirés sur un papier dont la couche d'émulsion était soigneusement enlevée pour que l'image proprement dite se trouve sur une feuille de papier photo très fine. Les têtes et les corps étaient ensuite découpés, probablement avec un scalpel, et assemblés avec beaucoup de soin. Les morceaux d'images étaient collés sur une photo qui servait d'arrière-plan, fixée sur un épais morceau de carton.

Le tout était retouché avec un pinceau et de la gouache. Des ombres étaient ajoutées pour que les éléments du montage semblent tous être éclairés par la même source de lumière, et tous les bords étaient travaillés de façon à être invisibles. Les parties de l'image dont on ne voulait pas étaient recouvertes de peinture, les personnages grossis ou amincis, on peignait des cheveux sur les têtes ou on les ôtait, la lumière était renforcée, etc.

Dans plusieurs montages, le texte était aussi peint directement. Cela se voit clairement dans le montage «Les bergers d'Arcadie » (fig. 11 et 12), où Lebrun, Daladier et Laval figurent dans le tableau Les Bergers d'Arcadie de Poussin. 


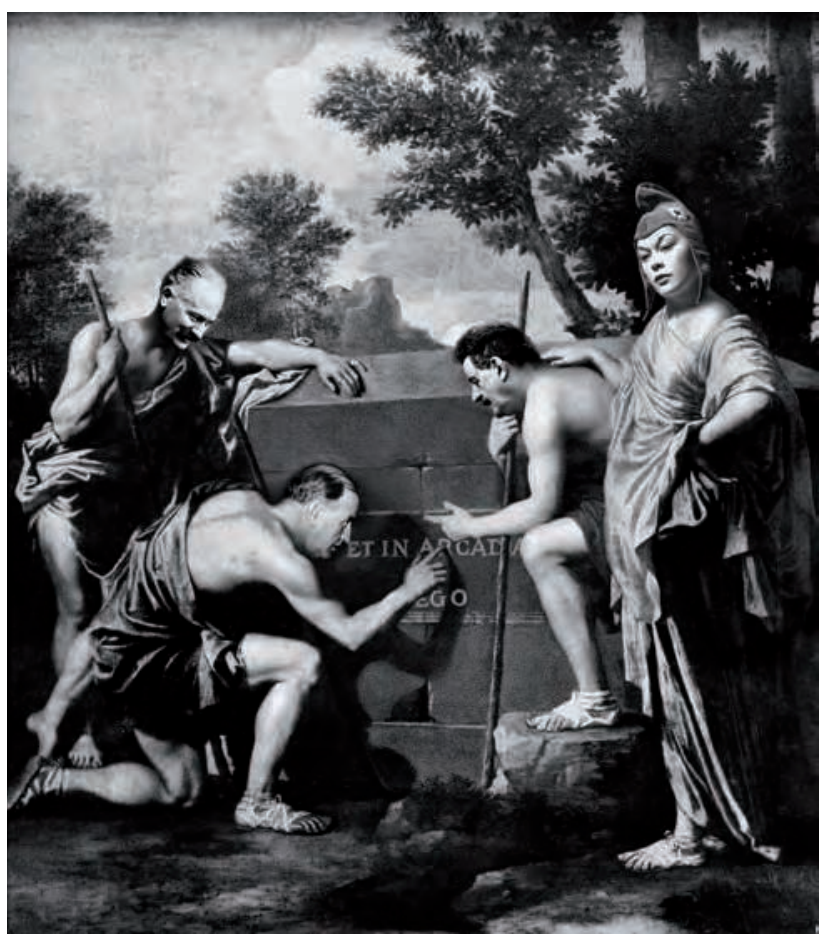

Fig. 11 : Marinus Jacob Kjeldgaard, « Les bergers d'Arcadie » Montage original $(23 \times 24,5 \mathrm{~cm})$ publié dans Marianne $\mathrm{n}^{\circ} 320$, 14 décembre 1938

De gauche à droite : Albert Lebrun, Édouard Daladier, Pierre Laval, Marianne

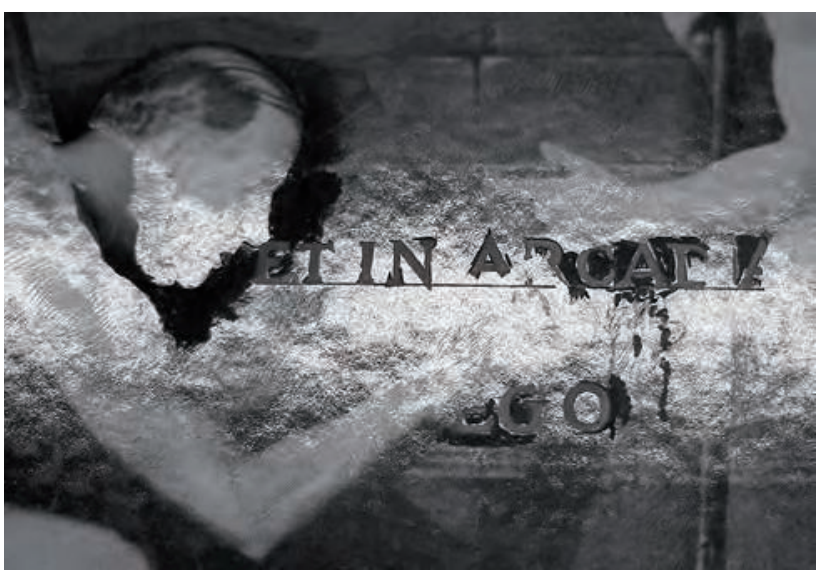

Fig. 12 : Retouche du montage « Les bergers d'Arcadie ». Les lettres sont écrites à la main. La part brillante de la photographie émane de l'original (tiré sur papier brillant). Les zones mates témoignent pour la plupart de l'utilisation d'une peinture qui ne réfléchit pas la lumière
Le mannequin russe très populaire Ludmila Feodoseyevna, qu'on appelle communément « Lud», a prêté son visage et son corps à la personne de Marianne, ce symbole de la République française. En l'espace de six mois, Marinus a fait appel à Ludmila à huit reprises.

À chaque fois, elle a respecté scrupuleusement les consignes qu'on lui a données et, comme c'est le cas ici, elle s'insère parfaitement dans l'image.

Le montage est maintenant terminé, reste à le photographier pour pouvoir en faire une copie prête à l'impression. Marinus s'y prend de deux façons : la plupart du temps, il fixe son montage sur un mur à l'aide de punaises, l'éclaire et fait une nouvelle photographie qui peut alors être copiée en autant d'exemplaires qu'il en a besoin sur le moment.

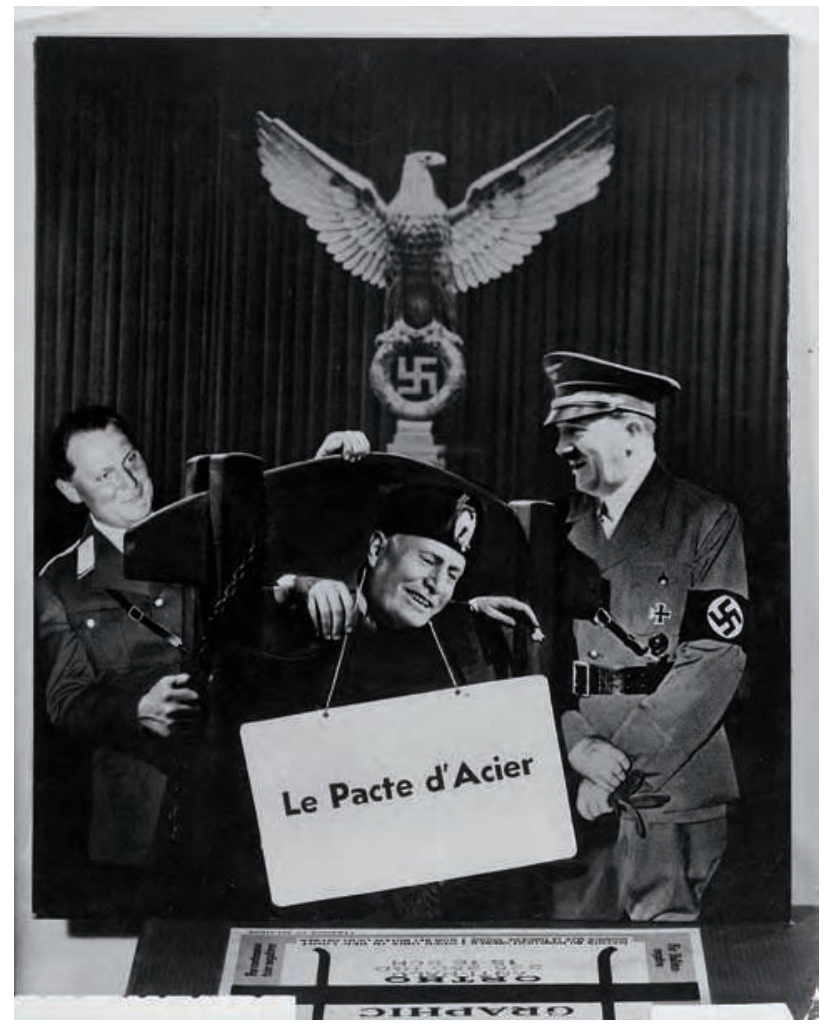

Fig. 13 : Marinus Jacob Kjeldgaard, « Le pacte d'acier»

Copie de photo d'après l'original $(30 \times 24,3 \mathrm{~cm})$, publié dans Marianne $\mathrm{n}^{\circ} 345,31$ mai $1939(23,9 \times 20,5 \mathrm{~cm})$

De gauche à droite : Hermann Göring, Benito Mussolini, Adolf Hitler 
À d'autres occasions, comme pour le «pacte d'acier » du 31 mai 1939 (fig. 13), il place simplement le montage sur un mur en le maintenant avec une boîte qui, à l'origine, avait contenu des films orthochromatiques. Il était inutile de chercher un système plus compliqué.

\section{La méthode d'impression}

Le premier numéro de Marianne paraît le 26 octobre 1932 sans aucune déclaration programmatique. Le seul lieu où l'on peut connaître les objectifs que s'était fixés le journal est une annonce de La Nouvelle Revue française du 1er octobre 1932, où l'on peut lire à la page 651 :

Les hebdomadaires littéraires sont à droite, ou le deviennent au 10 e numéro.

La pensée de la France est à gauche.

Nous voulons faire cesser cette contradiction.

C'est pourquoi nous créons MARIANNE.

Nous voulons qu'elle soit vivante et qu'elle soit vraie, qu'elle ne respecte ni les idées toutes faites ni les pouvoirs établis. C'est pourquoi nous l'appelons MARIANNE.

La vie moderne, c'est l'image. Nous voulons que MARIANNE soit abondamment et clairement illustrée.

C'est pourquoi MARIANNE appliquera à la reproduction de ses photographies une technique sans exemple dans le journalisme.

Cette nouvelle technique «sans exemple » était l'impression offset, une technique que Marianne fut le premier journal à utiliser en France. Cette technique permettait d'utiliser plusieurs sortes d'illustrations, de réaliser la mise en page plus rapidement et d'imprimer des photos de grande qualité. Alors que les journaux d'aujourd'hui impriment des photos de 85 lpi, les montages de Marianne avaient une résolution de 104 lpi. La qualité du papier était en outre bien meilleure qu'aujourd'hui, si bien que les montages et les photos étaient reproduits avec précision et une grande richesse de nuances.

Mais cette nouvelle technique créa de nombreuses difficultés à la rédaction. Il fallut inventer des procédures et des solutions techniques nouvelles, ce qui retarda la première publication de trois semaines. Mais une fois le journal lancé, on n'y trouva que des avantages, et aujourd'hui, quatre-vingts ans plus tard, les images sont encore nettes et agréables à regarder.

\section{Le processus créatif}

Nous ne savons pas grand-chose de la façon dont les idées germaient, mais nous savons que Marinus a été un collaborateur régulier du journal de 1932 à 1940, si bien que nous avons des raisons de penser qu'il a joué un rôle central dans son élaboration.

L'une des sources qui peut nous aider est l'article du The Strand Magazine de mars 1940 rédigé par le journaliste anglais H.J. Greenwall, mais l'on ne peut être sûr qu'il reflète la vérité. J. Greenwall est enthousiasmé par Marinus et ne le cache pas.

Il raconte par exemple à la page 457 :

Il est intéressant de voir comment Marinus travaille. C'est un mélange de journaliste et d'artiste. C'est avant tout le journaliste en lui qui décide quelle « histoire » il veut raconter. Dans la partie journalistique du cerveau du Danois jaillit l'idée qu'il faut faire quelque chose pour expliquer dans quelle mesure Hitler aime recevoir de mauvaises nouvelles [...] «Ah! dit la partie artistique du cerveau, je le tiens ! » Et un tableau de Lecomte du Nouÿ apparaît, bien entendu «Les porteurs de mauvaises nouvelles ». Ceux qui apportent de mauvaises nouvelles se font couper la tête.

Et un peu plus loin sur la même page :

Marinus fait pratiquement tout son travail dans le studio photo de Marianne, aux Champs-Élysées, mais il en exécute une partie chez lui, sur la rive gauche de la Seine.

H. J. Greenwall semble n'avoir aucun doute quant à la forte implication de Marinus dans la phase d'invention et dans la réalisation pratique des montages. Mais il n'était évidemment pas seul. Ses collaborateurs de Marianne, hautement qualifiés, lui ont sûrement fait des propositions et donné de bonnes idées, mais nous sommes incapables de préciser les apports respectifs.

Les seules traces avérées laissées par le travail de création se trouvent au dos des montages. Sur une grande quantité d'entre eux, on trouve une légende, les noms des personnages représentés et éventuellement des agences de photos ayant fourni les clichés. On reconnaît l'écriture de Marinus. 

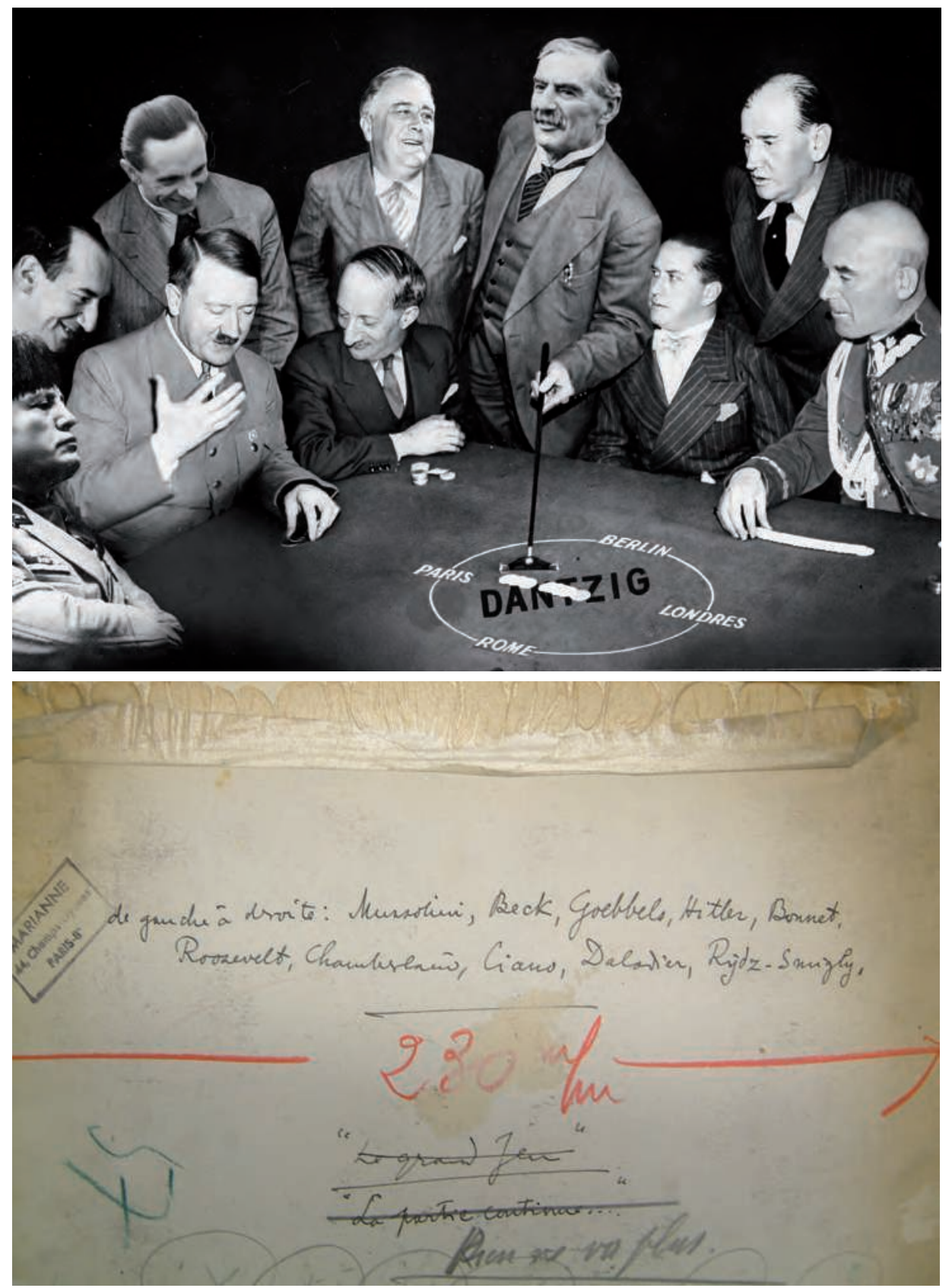

Fig. 14 et 15 : Marinus Jacob Kjeldgaard, «Rien ne va plus » (recto et verso)

Ce montage n'a jamais été publié, mais il est probable qu'il aurait dû l'être en août 1939, au moment de l'aggravation de la crise de Dantzig. On dispose, pour ce montage inédit, de l'original et d'une copie de photo d'après l'original $(24,7 \times 34 \mathrm{~cm})$ 
Prenons deux exemples. Au dos du montage inédit « Rien ne va plus » (fig. 14 et 15), on trouve, en plus des noms des personnages, trois propositions de légende. Deux d'entre elles sont rayées : « Le grand jeu » et « La partie continue... », mais l'inscription qui a été retenue est écrite en gras au crayon : «Rien ne va plus ». Les trois légendes sont écrites de la main de Marinus. Il apparaît clairement que l'on a hésité entre plusieurs légendes, avant que la plus habile ne s'impose. Nous ne savons pas exactement quand ce montage aurait dû être utilisé, mais sans doute en août 1939, quand Hitler exerce une pression sur la Pologne et Dantzig et fait monter la tension. Les légendes qui ont été rayées auraient laissé entendre que le jeu continuait, que tout espoir n'était pas perdu, tandis que la dernière dit clairement que les jeux sont faits. Les politiciens impuissants ne feront plus d'efforts et Hitler peut continuer à arborer un sourire satisfait.

Le 28 février 1940, dans le numéro 384 de Marianne, on voit en première page un montage qui représente Hitler et Staline dans la sculpture de Jean Turcan L'Aveugle et le paralytique (fig. 16). Le titre apparaît sur le montage luimême et toute autre légende est superflue. Fait curieux, une autre légende a malgré tout été écrite au dos (fig. 17) par Marinus, mais elle est en anglais. Comme Marinus a indiqué par ailleurs qu'il fournissait des montages à des magazines américains, et comme nous savons qu'il a effectivement donné des images à Life Magazine, on ne peut pas exclure que ce montage ait justement été destiné à être imprimé dans un pays anglophone.

Marinus a écrit : «The Blind carries the lame» (L'aveugle porte le paralytique) et au-dessous : «No straight and narrow path is theirs » (Leur chemin n'est ni droit ni étroit). Il se peut que cette dernière phrase ait été prévue comme légende. Elle fait allusion à l'Évangile selon saint Matthieu 7, 14 : «Mais étroite est la porte, resserré le chemin qui mène à la vie, et il y en a peu qui les trouvent. »

Le sens de cette citation biblique est clair : les deux hommes ne marchent pas sur le droit chemin, sur la voie étroite de la vertu, mais sont sur le chemin qui mène directement à la perdition, loin de la vie. C'était une affirmation prophétique, mais l'autre légende, c'est-à-dire le titre de la sculpture, était assez raffinée pour correspondre à la situation du moment, avec les deux conjurés handicapés qui s'aident mutuellement à avancer. C'est cette légende qui fut retenue.

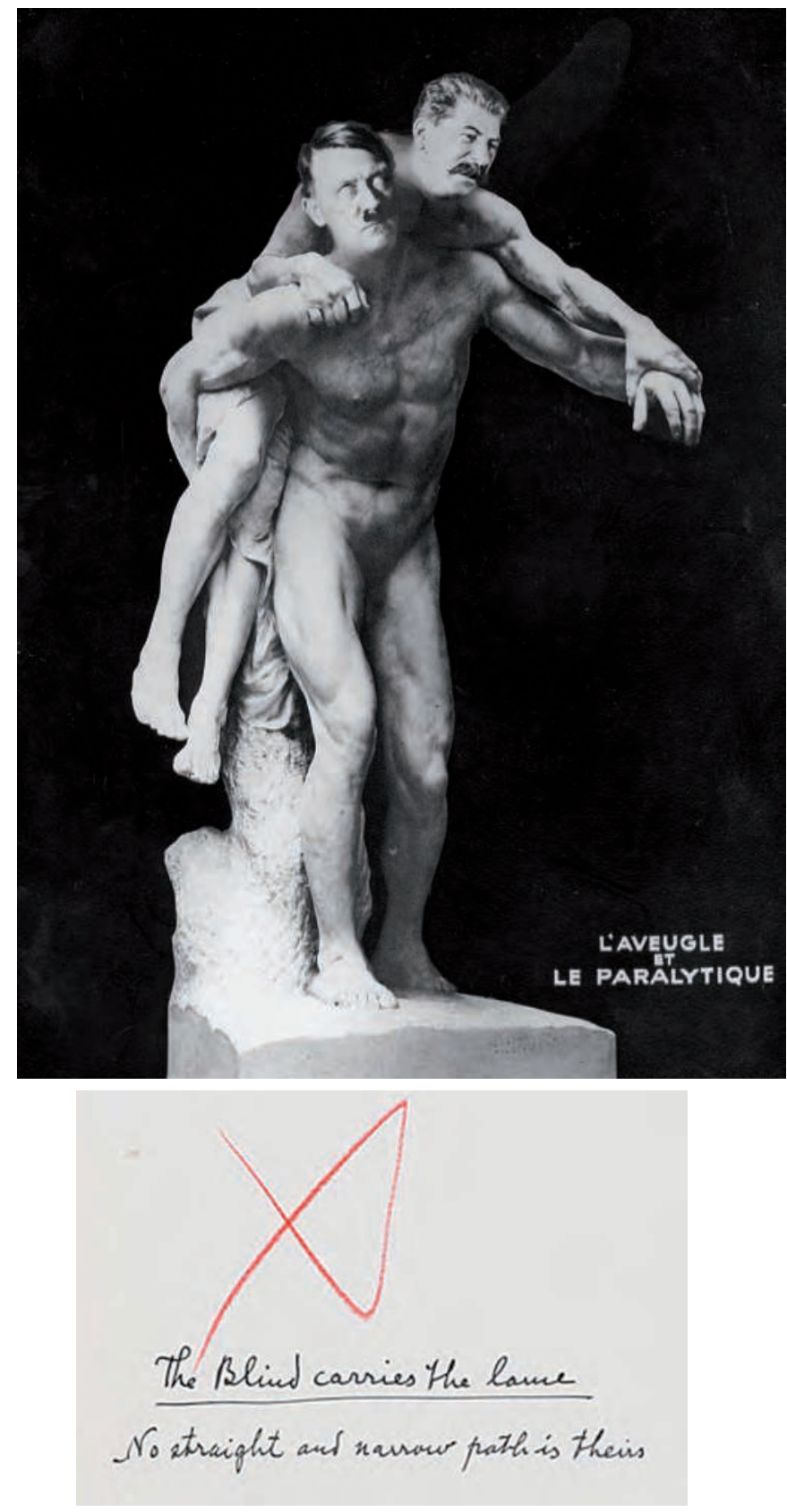

Fig. 16 et 17 : Marinus Jacob Kjeldgaard, «L'aveugle et le paralytique ». Copie de photo d'après l'original publié dans Marianne $\mathrm{n}^{\circ} 384$, 28 février $1940(28 \times 20,7 \mathrm{~cm})$ 


\section{L'héritage de Marianne}

On ne peut pas parler d'un héritage direct de Marianne. Le journal fut oublié par la France officielle aussitôt après la guerre, peut-être parce qu'il rappelait le fait qu'il y avait toujours eu une voix forte l'ayant mis en garde contre Hitler et ses compagnons assoiffés de guerre, dès 1933 et jusqu'à ce qu'il soit trop tard. Mais personne ne l'avait écoutée, et il n'était sans doute pas agréable de se le faire rappeler.

Il est pourtant clair que le journal était respecté, car les Français l'ont conservé. Même si cela fait maintenant plus de quatre-vingts ans que le premier numéro est sorti, on retrouve sans cesse de nouveaux exemplaires dans les caves et les greniers; on les vend sur eBay et d'autres sites d'enchères. Cela témoigne d'un amour pour la parole écrite et... pour Marianne.

\section{Post-scriptum}

\section{Quand le détective est dépassé : dix sujets de recherche!}

Malgré une recherche de plusieurs années, il reste encore un bon nombre de questions non résolues et plusieurs pistes qui ne mènent apparemment nulle part. Je saisis cette occasion pour énumérer dix d'entre elles.

1. Un certain Raymond Hamet, l'un des amis les plus proches de Marinus, était domicilié au 14, rue de la Glacière et avocat honoraire de la Cour d'appel. Il aurait possédé un tableau peint par Marinus, mais, jusqu'à présent, nous n'avons pas retrouvé trace ni de ses descendants, ni du tableau en question. Ils sont certainement quelque part, mais où ?

2. Marinus a précisé qu'il avait vendu trois de ses montages au bureau de la propagande, qui avait fait de l'un d'entre eux (Hitler comme peintre en bâtiment, Marianne, $\mathrm{n}^{\circ} 363$ ) un tract sur lequel figurait un texte en allemand, et dont, selon lui, deux cent mille exemplaires furent lâchés d'un avion sur l'Allemagne. Ce tract est d'ailleurs reproduit dans l'ouvrage de Klaus Kirchner, Propagande par tracts aéroportés pendant la seconde guerre mondiale (1982, vol. III, p. 233-234), mais il n'a pas été possible de retrouver l'original. Klaus Kirchner indique qu'il se trouve à la Bibliothèque nationale, mais celle-ci n'a pas été en mesure de le confirmer. Qui pourra le trouver?

3. Dans les documents déposés aux Archives nationales danoises (Rigsarkivet), il est indiqué que les affaires de Marinus furent vendues après sa mort pour 475,05 francs : on peut penser que cette succession comprenait aussi les montages qui ne firent leur apparition sur le marché aux puces de Vanves qu'au cours des années soixante-dix. Deux collectionneurs y virent des centaines de montages et en achetèrent chacun trente ou quarante exemplaires. Le vendeur prétendit la semaine suivante qu'ils avaient tous été brûlés dans l'incendie d'une camionnette, mais personne n'a jamais pu le confirmer. Il est donc possible qu'il existe quelque part plusieurs centaines de ces montages, mais où ?

4. Dans le rapport établi par le consul lors de la pose des scellés à l'appartement de Marinus, il est indiqué que Marinus était semble-t-il en rapport avec plusieurs maisons d'édition au sujet d'une couverture de livre en couleurs qu'il devait leur fournir. Mais de quelle maison d'édition s'agit-il, et de quels livres ?

5. Dans une lettre adressée à sa famille en 1945 , Marinus raconte la chose suivante : «Plusieurs de mes compositions ont été publiées dans la presse américaine. Hitler comme peintre en bâtiment, a été imprimé dans 140 journaux et revues américains, selon mon agent à New York. » Mais de quels journaux et de quelles revues s'agit-il, et de quel agent ? Et pouvons-nous réellement avoir confiance en lui ?

6. Le dessinateur danois Hans Bendix rencontre Marinus à Paris en 1921 ; ce dernier lui déclare qu'il est le rédacteur parisien d'une revue de Buenos Aires, mais quelle était cette revue, et pouvons-nous là aussi avoir confiance en lui ?

7. En 1984, le même Hans Bendix indique dans une lettre au neveu de Marinus, Niels Ole Kjeldgaard, qu'il 
avait « rencontré chez [Marinus] Fernand Léger et Marcel Leonie [sic ! Il s'agit de Marcelle Léoni], dont van Dongen a peint un portrait excellent et célèbre ». À cette période, l'artiste peintre danoise Fransisca Clausen semble même avoir eu une liaison très étroite avec Fernand Léger, mais on ne trouve aucune mention de Marinus dans les papiers qu'elle a laissés. Il se peut qu'il y ait des traces de Marinus dans les papiers laissés par Fernand Léger.

8. Et que faisait Marinus dans les années vingt ? Il prétend qu'il était correspondant et journaliste, mais où sont les preuves?

9. En 1948, Marinus a été photographié à Paris avec son neveu Per Frederik et un homme qui semble avoir été sculpteur, mais qui était cet homme, et à quel endroit était-ce ? Au dos de la photo, le neveu a écrit «Caszantous Cantous », mais qui est-ce ? Ce pourrait être par exemple Pierre Alfred Cazaubon (1885-1979) - mais est-ce exact?

10. Le 9 octobre 1945, Marinus se fait enregistrer à la Chambre des Métiers, qui lui attribue le numéro 138164.
Son numéro de téléphone est : ODE 8033. En 1954, une entreprise de photographie, Lynxa, est fondée avec le même numéro de téléphone, au 69 de la rue Froideveaux. Elle a existé jusqu'en 1969. Dans une lettre adressée à sa famille à l'automne 1957, son neveu Niels Ole Kjeldgaard raconte qu'il a passé une journée avec Marinus : «Il a vendu l'atelier photo - il a été trompé par un juif qui lui a fait perdre 1 million, et maintenant, il possède une agence de presse $^{1}$ qui occupe 4 photographes. Il écrit par ailleurs pour quatre journaux de province américains. » Dans quelle mesure tout cela peut-il être confirmé ? Et peut-on être absolument sûr que cela soit vrai ?...

Il y a donc encore du travail pour un détective entreprenant. Il suffit de sortir sa loupe!

Traduit du danois par Marc Auchet

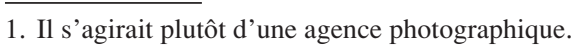


GUNNER Byskov est, depuis 1981, directeur d'études au Media College Denmark (Viborg). Il a été commissaire de plusieurs expositions sur Marinus Kjeldgaard (à Aarhus, Odense, Paris, Cologne...) ; il est l'auteur de Marinus. Karikaturtegner med kamera (Universitetsforlag, 2007); Hitler Blind, Stalin Lahm. Marinus und Heartfield : Politische Fotomontage der dreißiger Jahre (avec Bodo von Dewitz, Steidl Verlag, 2008); Marinus et Marianne. Photomontages satiriques (1932-1940) (Alternatives, 2008).

byskov@medieskolerne.dk

Résumés

Enquête sur les photomontages politiques de Marinus Jacob Kjeldgaard (1932-1940)

Le mystère prend forme en 2003, et il a fallu un vrai travail de détective pour l'éclaircir : qui était Marinus Jacob Kjeldgaard? Mais la réponse n'a fait naitre que plus de questions : quelle fut son histoire ? Pourquoi a-t-il quitté Copenhague pour Paris ? Pourquoi est-il tombé dans l'oubli ? Marinus a commencé son travail de photomontage dans J'ai vu en 1917, a été journaliste dans les années vingt et engagé en 1932 par Gaston Gallimard pour réaliser des caricatures photographiques en guise d'éditoriaux dans l'ambitieux hebdomadaire Marianne. Il fit jusqu'à l'occupation allemande en 1940 deux cents unes de Marianne et Marianne Magazine - pour être totalement oublié pendant soixante ans. Comment fallait-il comprendre ces montages ? Ils commentaient très précisément, avec humour et finesse, les questions de politique intérieure et, avec mordant, les mouvements fascistes européens - en particulier Hitler et son rêve de dominer le monde. Marianne et Marinus ont uni leur voix contre Hitler dès 1933, seuls ou presque à le faire à cette époque en France - ce qui expliquerait pourquoi il a paru commode d'oublier ces photomontages...

The mystery started in 2003, and substantial detective work was required to find the man named Marinus Jacob Kjeldgaard. But finding him only led to more questions. What was his story? Why did he move from Copenhagen to Paris? Why was he forgotten? He started making photomontages in $J^{\prime}$ ai $v u$ as early as 1917 , then worked as a journalist in the twenties and in 1932 was hired by Gaston Gallimard to make photographic caricatures as editorials in the ambitious weekly newspaper Marianne. He made 200 covers for Marianne and Marianne Magazine, until the German occupation in 1940 - and yet he was totally forgotten and his work remained unknown for over sixty years. How were his montages to be understood? They were subtle, humorous and precise comments on domestic policies, as well as sharp comments on the European fascist movements - in particular Hitler and his dream of ruling the world. Marianne and Marinus started raising their voices against Hitler as early as 1933, among the rare voices to do so at the time in France. Perhaps that was why it was convenient to forget all about these photomontages after the war...

Das Geheimnis nimmt im Jahre 2003 Gestalt an, und es bedurfte einer wahren Detektiv-Arbeit, um es aufzuklären: Wer war Marinus Jacob Kjeldgaard? Doch die Antwort hat zusätzlich weitere Fragen nach sich gezogen: Was war seine Geschichte? Warum verließ er Kopenhagen, um in Paris zu leben? Warum ist er in Vergessenheit geraten? Marinus begann seine Arbeiten im Bereich der Photomontage in J'ai vu im Jahre 1917, und er war in den 1920er-Jahren Journalist; 1932 wurde er von Gaston Gallimard engagiert, um photographische Karikaturen in der Art von Editorial-Beiträgen in der ambitionierten Wochenzeitschrift „Marianne“ zu realisieren. Bis zur deutschen Okkupation im Jahre 1940 wirkte er an 201 Ausgaben von Marianne und Marianne Magazine mit, um dann aber 60 Jahre lang ganz in Vergessenheit zu geraten. Wie soll man seine Montagen verstehen? Sie kommentierten sehr präszise, humorvoll und feinsinnig Fragen der Innenpolitik und, mit Schärfe, die europäischen faschistischen Bewegungen - insbesondere Hitler und seinen Traum, die Welt zu beherrschen. Gemeinsam haben Marianne und Marinus seit 1933 ihre Stimme gegen Hitler erhoben, fast als einzige zu dieser Zeit in Frankreich - das erklärt, warum es als bequem erschien, jene Photomontagen zu vergessen...
El misterio se planteó en 2003 y fue necesario un verdadero trabajo de detective para resolverlo: ¿quién era Marinus Jacob Kjeldgaard? La respuesta hizo surgir nuevos interrogantes: ¿cómo fue su historia?, ¿por qué abandonó Copenhague para instalarse en París?, ¿por qué cayó en el olvido? Marinus comenzó su trabajo de fotomontaje en $J$ ' ai vu en 1917, se desempeñó como periodista durante los años veinte y en 1932 fue contratado por Gastón Gallimard para realizar caricaturas fotográficas, a modo de editoriales, en el ambicioso semanario Marianne. Hasta el momento de la ocupación alemana, en 1940, realizó doscientas portadas de Marianne y Marianne Magazine, cayendo luego en el olvido durante sesenta años. ¿Cómo debían ser entendidos esos montajes? Como comentarios precisos -plenos de humor y de fineza- de cuestiones de política interior, y mordaces con los movimientos fascistas europeos - en particular, Hitler y su sueño de dominar el mundo. Marianne y Marinus fueron, durante ese periodo, los únicos en Francia-o, casi- que unieron sus voces contra Hitler desde 1933, razón por la cual resultó cómodo olvidarse de esos fotomontajes...

Tutto iniziò come un mistero nel 2003, e c'è voluto un vero e proprio lavoro da detective per rintracciare un uomo chiamato Marinus Jacob Kjeldgaard. Quando lo trovai, altri misteri spuntarono: quale fu la sua storia? Perché lasciò Copenaghen per trasferirsi a Parigi? Perché è stato dimenticato? Marinus cominciò a realizzare fotomontaggi per $J$ ' ai vu nel 1917. Giornalista negli anni '20, nel 1932 fu assunto da Gaston Gallimard con il compito di realizzare caricature fotografiche in forma di editoriali per l'ambizioso settimanale Marianne. Nonostante abbia realizzato duecento prime pagine di Marianne e Marianne Magazine fino all'occupazione tedesca del 1940, Marinus Jacob Kjeldgaard venne completamente dimenticato e il suo lavoro rimase sconosciuto per oltre sessant'anni. Che significato avevano i suoi montaggi? Commentavano con sottigliezza, humour e precisione i problemi di politica interna e con determinazione i movimenti fascisti europei - in particolare Hitler e il suo sogno di dominare il mondo. Marianne e Marinus iniziarono ad alzare le loro voci contro Hitler nel 1933, in un periodo in cui in Francia non si sentivano molte voci analoghe. Tutti sembravano pensare a qualcos'altro - e forse ciò spiega perché, nel dopoguerra, fu comodo dimenticare quei fotomontaggi.

O mistério adensou-se em 2003 e só com bastante trabalho de detetive foi possível chegar a um homem chamado Marinus Jacob Kjeldgaard, e com ele a uma nova série de questões: qual era a sua história? O que o trouxe de Copenhagen para Paris? Porque foi esquecido? Já em 1917 fazia montagens para $J^{\prime}$ ai vu, nos anos vinte trabalhou como jornalista e em 1932 foi contratado por Gaston Gallimard para fazer caricaturas fotográficas como comentário editorial no ambicioso semanário Marianne. Foi autor de duzentas capas para esse jornal e para Marianne Magazine, até que a ocupação alemã em 1940 pôs fim a tudo - e o lançou no esquecimento por mais de sessenta anos. As suas montagens eram comentários sofisticados e bem humorados, com opiniões claras sobre política interna e críticas afiadas aos movimentos fascistas da Europa de então, a começar por Hitler e os seus sonhos de domínio mundial. Poucas vozes em França, nos anos trinta, se manifestaram contra Hitler como Marianne e Marinus, o que talvez ajude a compreender porque se tornou cómodo esquecer estas fotomontagens. 